\title{
F-response frequency in motor neuron disease and cervical spondylosis
}

\author{
S PEIOGLOU-HARMOUSSI, ${ }^{*}$ P R W FAWCETT, ${ }^{*}$ D HOWEL, $\dagger$ D D BARWICK* \\ From the Department of Clinical Neurophysiology, ${ }^{*}$ Regional Neurological Centre, Newcastle General Hospital, \\ and the Department of Medical Statistics, $\uparrow$ University of Newcastle upon Tyne, UK
}

SUMMARY During a train of 200 stimuli, F-response frequency, frequency of identical responses, and F-response shape were studied in the ulnar nerve of 17 patients with motor neuron disease (MND) and 16 patients with cervical spondylosis (CS). In MND patients, F-response frequency varied between $5 \%$ and $96 \%$ with a median of $39 \%$ which was significantly lower than controls $(\mathrm{p}<0.001)$, and showed a significant rank correlation with the $\mathrm{M}$-response amplitude $(\mathrm{r}=0.62$, $\mathrm{p}<0.004$ ). Identical responses occurred more frequently than in controls (median $30.8 \%$, $\mathrm{p}<0.001$ ). F-response frequency was normal in CS patients (median $76.3 \%$, range $35 \%$ to $97 \%$ ), but the frequency of identical responses (median 6.3\%) was higher than normal ( $p<0.01$ ). Reduced F-response frequency in MND was thought to reflect loss of lower motor neurons, while the presence of spacticity was probably the major factor underlying the increased frequency of identical responses in both disorders. F-response shape tended to be simpler in MND and rather more complex in CS patients than controls.

Recent studies in normal human subjects have shown the production of F-responses in individual motor neurons to be an uncommon event, ${ }^{12}$ whereas in the motor neuron pool as a whole such recurrent responses occur relatively frequently. ${ }^{3}$ The production of these responses seems to depend mainly on the balance of excitatory and inhibitory activity at the appropriate spinal cord level. ${ }^{1}$ In general, increased levels of excitability as seen in spasticity, lead to an increase in F-response production by responding motor neurons. ${ }^{1}$ On the other hand frequency falls in the immediate postictal period following a cerebrosvascular accident during which muscle tone is decreased and the deep tendon reflexes are depressed. 4

It might also be expected that F-response frequency may be influenced by changes affecting the lower motor neuron, and for example, that a reduction in their number might lead to a decrease in F-response

†Present address: B Department of Neurology, Central Hospital, Saint Dimitrios, Salonica, Greece.

Address for reprint requests: Dr PRW Fawcett, Department of Clinical Neurophysiology, Regional Neurological Centre, Newcastle General Hospital, Westgate Road, Newcastle upon Tyne NE4 6BE, UK.

Received 7 February 1986 and in revised form 16 June 1986. Accepted 23 June 1986 production from the motor neuron pool.

With these points in mind F-response frequency has been studied in patients with motor neuron disease (MND) and cervical spondylosis (CS) in whom variable combinations of upper and lower motor neuron changes may be seen. In addition to the overall frequency of F-response production, the frequency with which identical F-responses occur and Fresponse shape have also been examined.

\section{Material}

\section{Motor neuron disease}

Seventeen patients, ( 15 males, 2 females) aged between 37 to 74 years (mean 59.1 SD 10.2) with a definite clinical diagnosis and electromyographic evidence of MND were studied. The duration of the symptoms varied between 6 to 38 months with a mean of 15.4 months. All but two of the patients were moderately to severely incapacitated by their illness, while only two showed no muscle wasting and eight had moderate to severe wasting of the hypothenar muscles from which the recordings were obtained. All but three cases had some degree of weakness of the appropriate muscles which was moderate to marked in 10 cases. Generalised hyper-reflexia was present in all of the patients.

\section{Cervical spondylosis}

The cervical spondylosis group contained 16 patients (14 male, 2 females) aged between 46 and 70 years (mean 57.6 SD 6.1). These patients were divided into two subgroups 
with eight in each according to the neurological and neuroradiological findings, one with predominantly cervical radiculopathy, the other with cervical myeloradiculopathy. Only one of the patients with cervical radiculopathy had slight wasting of the hypothenar muscles although all had mild to moderate weakness and six had absent or inverted reflexes in the same upper limb. In the myeloradiculopathy group five had slight to moderate muscle wasting, all had moderate to severe muscle weakness and seven had abnormally brisk reflexes in the same limb.

The control group consisted of 21 healthy subjects ( 14 males, 7 females) aged between 30 and 66 years (mean 48 SD 10), the data from whom have been presented elsewhere. $^{3}$

\section{Methods}

The subjects lay on a couch with the arm and hand outstretched in the supine position. A train of 200 supramaximal stimuli was applied at a frequency of $1 \mathrm{~Hz}$ to the ulnar nerve at the wrist using bipolar surface electrodes (cathode proximal) and a pulse width of $0.2 \mathrm{~ms}$. Bellytendon recordings were made from the hypothenar muscles using surface saline soaked pads of $1 \mathrm{~cm}$ diameter connected to silver disc electrodes mounted in a plastic frame, taking care to ensure the active electrode was placed over the endplate zone. A sweep speed of $5 \mathrm{~ms} / \mathrm{div}$ and gain of $0.2 \mathrm{mV}$ or $0.5 \mathrm{mV} / \mathrm{div}$ were used. The subject was encouraged to relax to prevent interfering volitional activity and the recording was carefully monitored for spontaneously occurring fasciculation potentials. All the studies were performed on a Medelec MS6 EMG apparatus (Medelec Ltd, Old Woking, England) and permanent recordings were made with a fibre-optic recorder using a paper speed of $5 \mathrm{~cm} / \mathrm{s}$ in raster mode. Only those deflections which had a clear deviation from the baseline and an amplitude of at least $40 \mu \mathrm{V}$ were accepted as F-responses in order to differentiate them from background noise.

For each F-response the latency to onset and peak-topeak amplitude were measured using transparent graph paper with $1 \mathrm{~mm}$ graticules and the shape was assessed in terms of the number of negative peaks which achieved an amplitude of $10 \mu \mathrm{V}$ or over. The series of responses was then examined for the presence of identical F-responses based on the latency and shape characteristics. When two or more responses were considered to be identical, the first was carefully copied onto tracing paper and this copy was superimposed on subsequent similar responses. They were accepted as identical responses if superimposition proved their shapes and amplitudes to be virtually the same. Slight variations in shape due to noise had to be taken into account in this respect.

The following parameters were then determined in each case. (1) Overall F-response frequency, which was defined as the percentage of 200 stimuli producing an F-response, (2) Frequency of identical responses: the following procedure was used in order to take into account the variable number of responses produced during the series of 200 stimuli in different subjects. The frequency with which each identical response occurred and the total number of different Fresponse shapes were determined. The identical responses were then sorted into subgroups according to the number of times they occurred that is, 2-5, 6-10, 11-15, 16-20 and $>20$. The number of responses in each subgroup was then determined and expressed as a percentage of the total number of different F-response shapes (see Pieoglou-Harmoussi et al $1985^{3}$ for more detailed explanation). Finally the percentages of different F-response shapes occurring (a) singly and (b) two or more times were calculated.

The data of each disease group were compared with controls using the Mann Whitney U-test. Correlations were assessed using Spearman's Rank Correlation test.

\section{Results}

\section{Motor neuron disease}

F-response frequency and frequency of identica responses The data concerning F-response free quency and frequency of identical responses i区 patients with motor neuron disease are presented if $\overrightarrow{0}$ table 1. The frequency of F-responses arising from the whole muscle varied considerably, ranging from a minimum of $5 \%$ to a maximum of $96 \%$ with a median of $39 \%$, which was significantly lower than in the controls ( $p<0.001$ ) (fig 1). The frequency of identical responses (table 1), measured in terms of the number of responses occurring two or more times, revealed a median of $30.8 \%$ (range 6-100\%) which was markedly and significantly higher than the control group median value of $3.3(p<0.001)$. Furthermore, while responses very rarely occurred more than 10 times per series of 200 stimuli in control subjects, many responses were seen up to 15 to 20 times (table

Table 1 F-response frequency during a train of 200 stimuli in the ulnar nerve in controls and patients with MND and CS

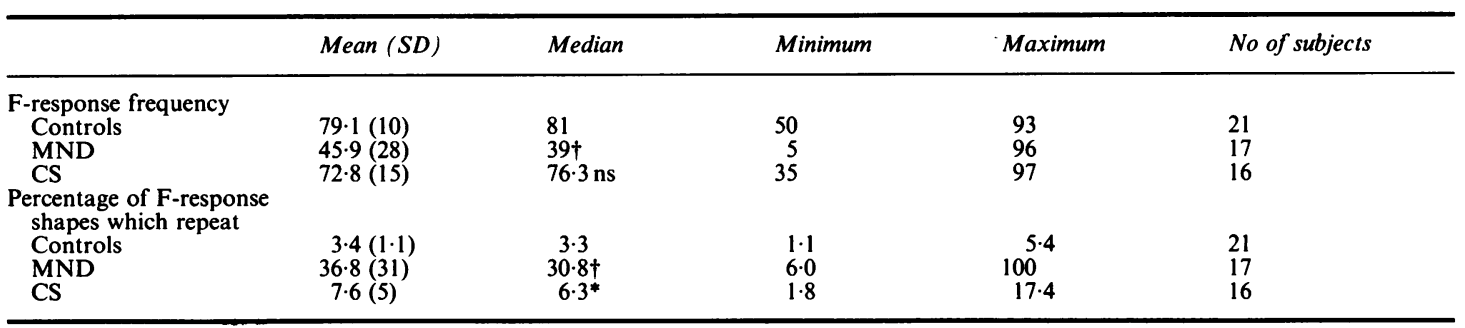

ns - Not significantly different from controls. * and + Significantly different from controls (Mann Whitney p $<0.01$ and 0.001 respectively). 

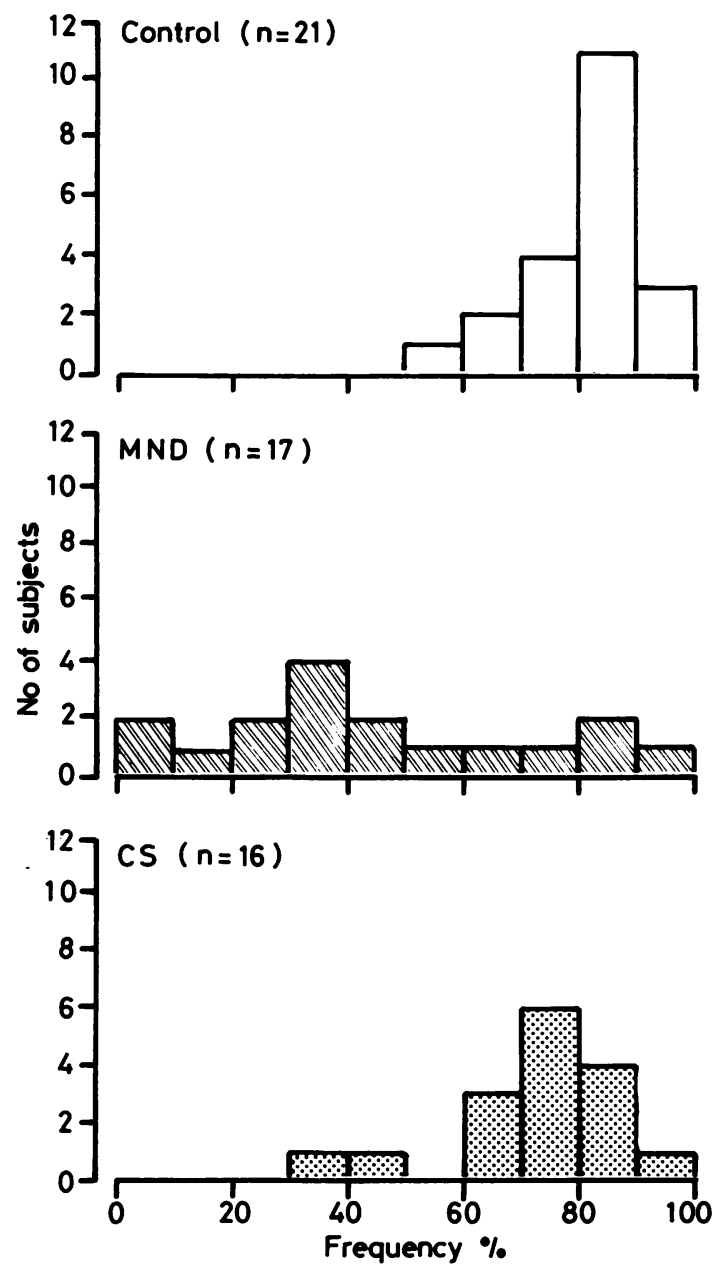

Fig I Distribution of F-response frequencies in controls, and patients with MND and CS. The MND median value (39\%) is significantly lower than the control median $(80.5 \%)(p=$ $<0.001)$. The CS median value $(76.3 \%)$ is not significantly different from the control value.
2), and the maximum number times an individual repeating response occurred was significantly increased with a median of 24 (range 2 to 60 ) in MND patients compared with controls (median 5 , range 2 to $11, \mathrm{p}<0.001)$.

The median amplitude of the evoked M-response was significantly reduced in comparison with controls (control $11.4 \mathrm{mV}$, range $8-17 \mathrm{mV}$; MND $4.5 \mathrm{mV}$, range $0.35-13.5 \mathrm{mV} ; \mathrm{p}<0.0001$ ) and a slight but significant positive rank correlation was found between $\mathrm{F}$-response frequency and size of the $\mathrm{M}$ response $(r=0.62, p=0.004)$, indicating that $F$ response production fell with the loss of lower motor neurons. At the same time there was some evidence that the frequency of repeating responses increased as the $\mathrm{M}$-response amplitude declined $(\mathrm{r}=0.56, \mathrm{p}=$ $0 \cdot 01$ ).

Figure 2 illustrates part of a recording from a 37 year old man with MND in which a total of $90 \mathrm{~F}$ response were produced during the complete 200 stimulus train, a frequency of $45 \%$ which is slightly below the lower limit of $50 \%$ observed in the control population. As the figure shows, there was only a small number of different F-response shapes, most of which occurred two or more times. In some traces, indicated by asterisks, it is possible to identify clearly two separate F-responses.

Even in those recordings in which the overall $\mathrm{F}$ response frequency was within the normal range, an increased proportion of the responses repeated two or more times. At the other extreme in the presence of severe muscle wasting only two, or in one case a single F-response was elicited. In this situation the response showed a marked increase in firing frequency.

F-response shape The shape of F-responses was rather less variable than seen in controls, with a greater tendency for simple shapes, particularly in the presence of moderate to marked muscle wasting when there were fewer responses. The distribution of numbers of negative peaks, based on 1000 responses, was positively skewed (fig 3 ), with a preponderance of single peaks and a maximum of 4 peaks in a small proportion.

Table 2 Frequency of identical F-responses occurring during a train of 200 stimuli in the ulnar nerve in controls and patients with $M N D$ and $C S$

\begin{tabular}{|c|c|c|c|c|c|c|c|}
\hline$N$ & 1 & $2-5$ & $6-10$ & $11-15$ & $16-20$ & $>20$ & $\begin{array}{l}\text { No of } \\
\text { subjects }\end{array}$ \\
\hline \multicolumn{8}{|c|}{$\begin{array}{l}\text { Mean percentage of } \\
\text { F-response shapes } \\
\text { occurring N times }\end{array}$} \\
\hline $\begin{array}{l}\text { Controls } \\
\text { MND } \\
\text { CS }\end{array}$ & $\begin{array}{l}96 \cdot 6 \\
61 \cdot 7 \\
92 \cdot 3\end{array}$ & $\begin{array}{r}3 \cdot 0 \\
14 \cdot 5 \\
5 \cdot 1\end{array}$ & $\begin{array}{l}0.3 \\
6 \cdot 4 \\
1.9\end{array}$ & $\begin{array}{l}0 \cdot 1 \\
3 \cdot 4 \\
0 \cdot 4\end{array}$ & $\begin{array}{l}0 \\
6 \cdot 5 \\
0 \cdot 1\end{array}$ & $\begin{array}{l}0 \\
7 \cdot 4 \\
0 \cdot 2\end{array}$ & $\begin{array}{l}21 \\
17 \\
16\end{array}$ \\
\hline
\end{tabular}


$S$

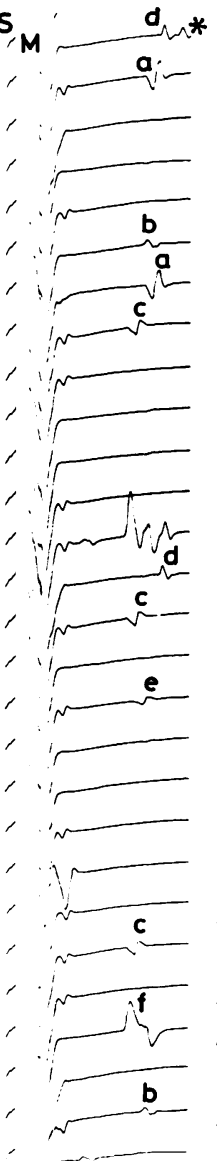

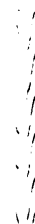
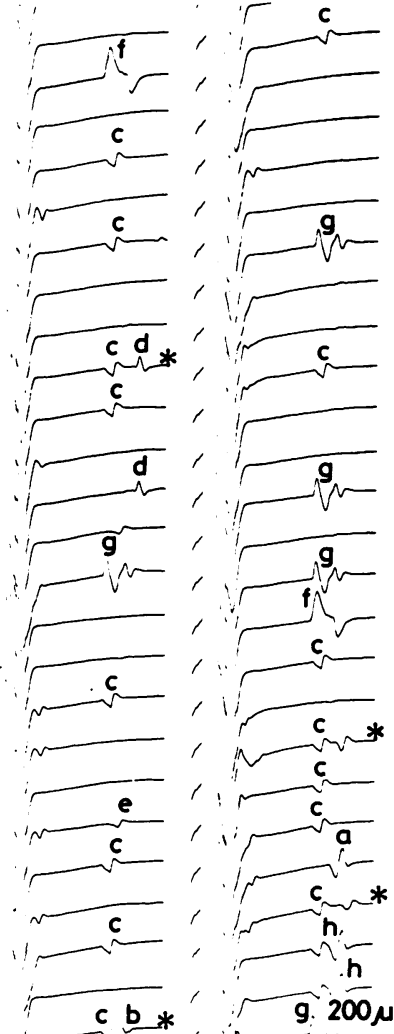

n. h

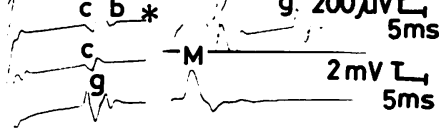

Fig 2 Sample of a recording of sequential F-responses obtained during a train of 200 supramaximal stimuli to the ulnar nerve at the wrist in a 37 year old man with MND of 6 months duration. Reduced $F$-response frequency (45\%).

Note high incidence of repeating responses (40\%) identified by letters. Two separate responses can be identified in some sweeps, indicated by asterisks.

\section{Cervical spondylosis}

In view of the small number of cases in each subgroup the cervical radiculopathy and myeloradiculopathy patients have been considered together, and only if clear differences between the two subgroups were found will they be commented upon. Data concerning F-response frequency and the frequency of identical responses are presented in tables 1 and 2 .

F-response frequency and frequency of identical responses The overall frequency of F-responses varied between $35 \%$ and $97 \%$ with a median of $76.3 \%$, which was not significantly different from the control
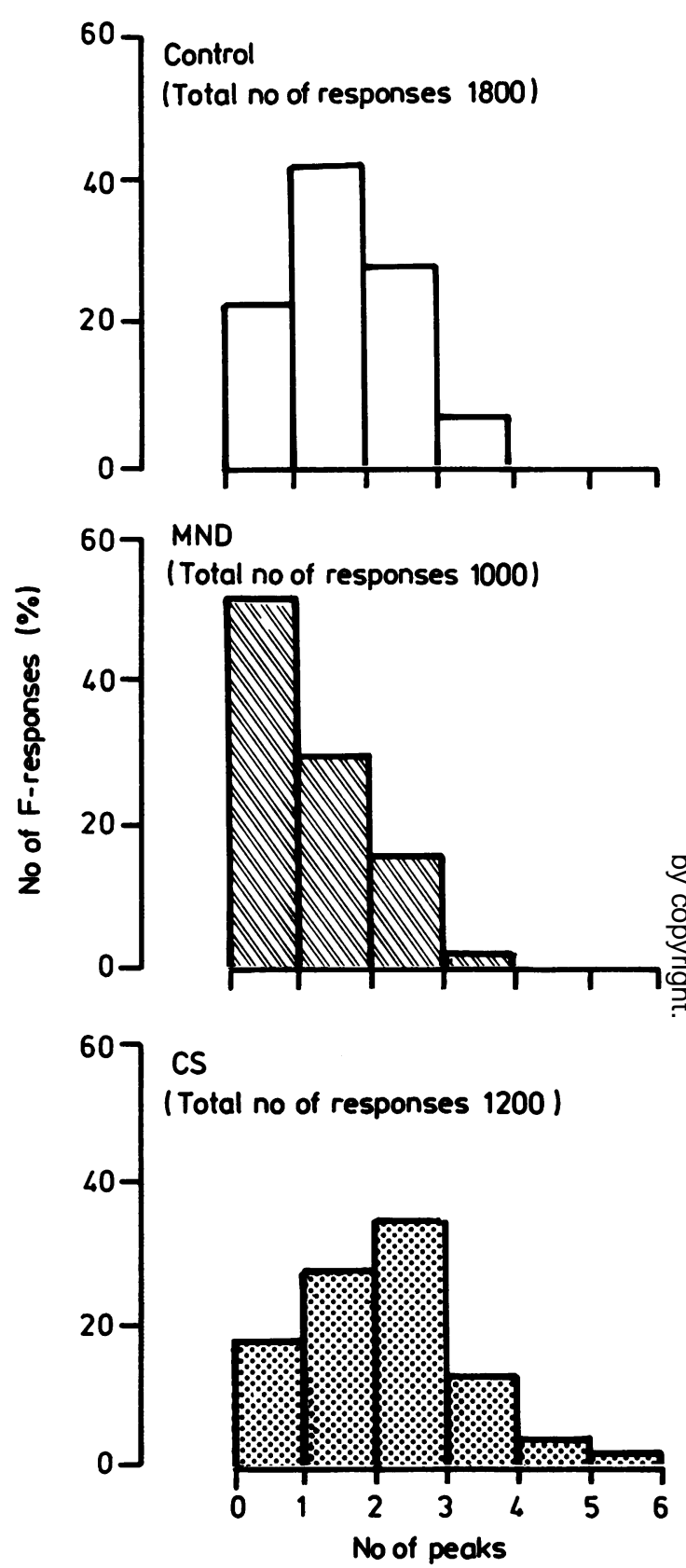

Fig 3 Histogram of the number of negative peaks in F-responses recorded from control subjects and patients with $M N D$ and $C S$.

population. However, a significantly larger number of identical responses was seen $(p<0.01)$, the median number of responses occurring two or more times 


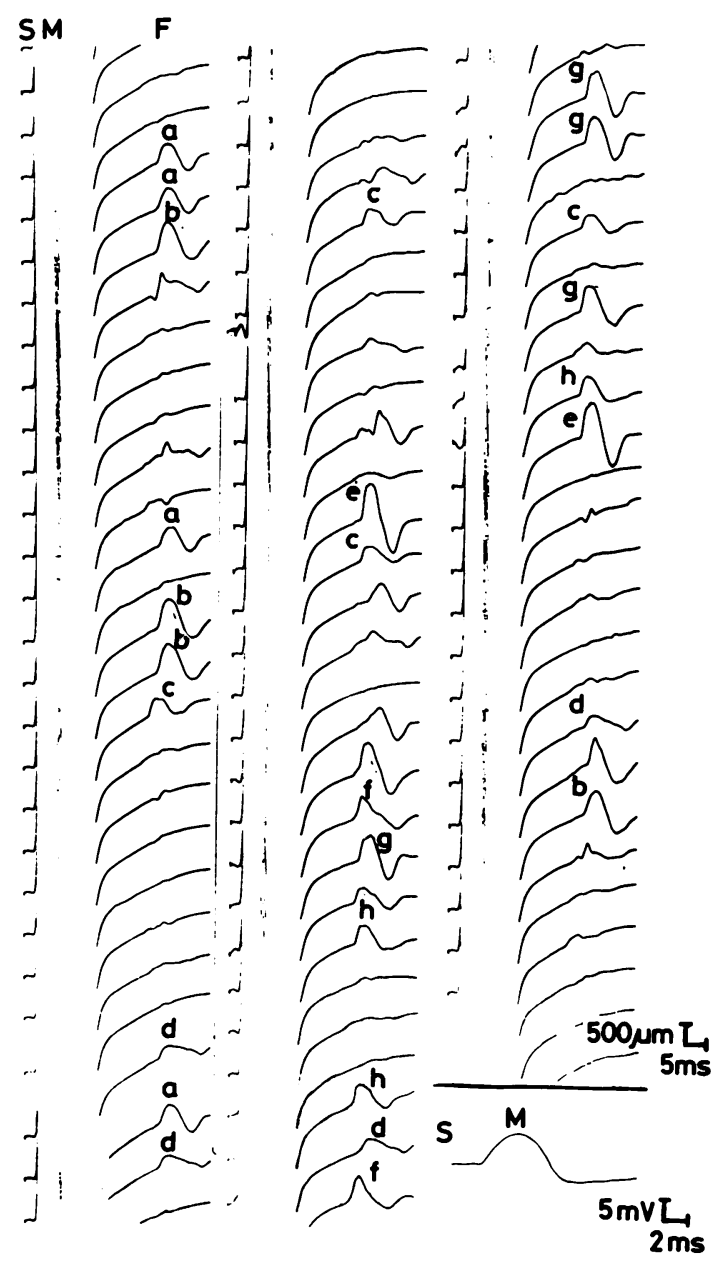

Fig 4 Sample of a recording of F-responses from the ulnar nerve in a 61 year old man with cervical myeloradiculopathy. Normal F-response frequency (69\%); increased frequency of repeating responses $(14 \%)$, identified by letters.

being $6.3 \%$ with a range of $1.8 \%$ to $17 \cdot 4 \%$. There was also an increase in the frequency with which each repeating response occurred, (fig 4 and table 2), the maximum number of times a response repeated ranging from 4 to 22 with a median of $10 \cdot 5$, which was significantly higher than in controls (median 5, $\mathrm{p}<0.001$ ).

The median F-response frequencies in the subgroups $\mathrm{CR}$ and $\mathrm{CMR}$ (77\% and $71 \%$ respectively) were not significantly different $(p>0.05)$. However there was some indication that the frequency of repeating responses was significantly different in the two groups, with median values of $5 \cdot 2 \%$ and $8.3 \%$ in CR and CMR respectively $(p=0.03)$.

The median amplitude of the M-response was slightly but significantly smaller than the control value (control $11.5 \mathrm{mV}$ range $8-17 \mathrm{mV}$; CS $10.0 \mathrm{mV}$ range $6-13 \mathrm{mV} ; \mathrm{p}=0.05$ ), but in contrast to the MND group there was no correlation between Fresponse frequency or frequency of repeating responses and the amplitude of the $\mathrm{M}$-response.

F-response shape The degree of variability of $\mathrm{F}$ response shape (based on 1200 responses) was similar to that seen in controls (fig 3), although some of the responses had more complex shapes with up to 6 negative peaks.

\section{Discussion}

Motor neuron disease is characterised by progressive degeneration of upper and lower motor neurons, leading to a combination of both spasticity and muscle wasting and weakness, the relative proportions of which may vary in different individuals. A similar combination of upper and lower motor neuron involvement may be encountered in patients with cervical myeloradiculopathy, although the changes are generally less pronounced than in MND which has a more rapid course and malignant outcome.

The distal muscles of the upper limb used for recording in the present study appear to be affected relatively early in the course of the disease in most patients with MND, ${ }^{5}$ although there may be considerable variability among individuals. Involvement of these muscles supplied by the C8-T1 segments is less marked in cervical myeloradiculopathy which generally affects the C5-C6 myotomes.

The major finding in patients with MND was of an overall reduction in F-response frequency compared with normal subjects, confirming the observations of Morimoto $^{6}$ in a smaller sample of cases. F-response frequency was also positively correlated with the amplitude of the $\mathbf{M}$-response. The latter is a reflection of the volume of active muscle and hence can be taken as a measure of the degree of muscle wasting. The relationship between $\mathrm{F}$-response frequency and $\mathrm{M}$ response amplitude suggests that the main cause for the reduction in F-response production is a decrease in the number of available lower motor neurons as they degenerate during the course of the disease. Nevertheless, despite a general reduction in frequency, there was a wide scatter of frequency values ranging from $5 \%$ to as high as $96 \%$ which is at the upper limit of normal. All but three of the patients in the present study had clinical evidence of weakness in the intrinsic muscles of the hand. According to Wohlfart, ${ }^{7}$ as a consequence of compensatory reinnervation, up to $30 \%$ of ventral horn cells may be lost before clinical symptoms become evident, so that some degree of neuronal fall out might be expected in the majority of the present cases. Consequently the presence of nor- 
mal F-response frequencies in some patients might at first sight appear surprising.

The explanation for this finding lies in the marked increase in number of identical responses. Thus in those cases with minimal involvement of the hypothenar muscles, loss of motor neurons is more than compensated for in terms of F-response production by an increase in frequency in individually responding neurons. The percentage of repeating responses was negatively correlated with the amplitude of the $\mathbf{M}$ response, suggesting an increasing tendency for individual motor neurons to produce F-responses as the disease progresses. Despite this, a stage is reached when the overall dropout of neurons eventually leads to a reduction in F-response frequency from the whole muscle. In a recent study Petajan ${ }^{8}$ found only a slight and insignificant increase in the number of repeating responses during a series of $20-40 \mathrm{~F}$ responses in the extensor digitorum brevis muscle in patients with amyotrophic lateral sclerosis. However, electromyographic examination of the muscles in the latter study showed normal or slightly reduced recruitment suggesting minimal loss of lower motor neurons, and this factor probably accounts for the difference in findings, since many of the present cases had clear and marked muscular atrophy.

One of the reasons for the increase in identical responses may be deduced from the single fibre EMG study on F-response frequency by Schiller and Stålberg ${ }^{1}$ in patients with spasticity. They found that while the proportion of motor neurons giving rise to F-responses did not change, there was a significant increase in the production of F-responses in responding neurons. A marked increase in the frequency with which each individual F-response occurred was also noted in the present study, with values as high as 20 and over in many cases.

However, in contrast to Schiller and Stålberg's ${ }^{1}$ findings there was also an increase in the proportion of neurons producing repeating responses in MND patients. While this probably represents a true increase in the number of neurons generating identical responses, another possible mechanism relating to the differences in recording arrangements has also to be considered. In the present study the use of surface recording electrodes precludes a one to one relationship between the F-response and individual motor units, as obtains in single fibre recordings. It has been shown in normal subjects ${ }^{3}$ that surface recorded F-responses may be composed of potentials from two or more motor units which have simultaneously generated late responses. Thus as motor neurons are lost, so the chances of overlapping diminish and repeating responses from individual motor units may be more easily recognised, resulting in an apparent rather than actual increase in the proportion of neurons producing identical responses.

F-response frequency was not significantly different from normal in the combined group of patients with CS. In the subgroup of cases with predominantly myelopathic features the range of values was wider than seen in controls, and although the mean value was slightly lower than normal, the difference was not statistically different. Nevertheless a significantly larger than normal number of identical responses was found, particularly in cases with myelopathy. The increase in identical F-responses was due to a greater number of different F-responses firing a few times rather than a marked increase in F-response repetition from a few motor units.

Amplitudes of M-responses recorded from the hypothenar muscles in the CS group were only slightly reduced compared with controls, indicating at most a minimal drop-out of lower motor neurons. Thus it is not surprising that there was an insignificant change in F-response frequency in these cases. However, the slight increase in identical responses, seen particularly in the myelopathy group, might imply increased excitability in the motor neuron pool secondary to upper motor neuron involvement. This may be combined with unmasking of repeating responses due to less overlapping of potentials from individual motor units in those cases where lower motor neurons have been lost.

Studies in patients with cervical myeloradiculopathy have shown corticospinal tract damage and anterior horn cell loss secondary to cord ischaemia. ${ }^{9}$ These changes are particularly striking in chronic cases and are most marked under the area of compression. ${ }^{10}$ As the balance of upper and lower motor neuron changes may vary in different patients, the influence on F-response production will differ accordingly. Thus in one patient with recent onset of spasticity but no evident muscle wasting an increased F-response frequency was recorded, while lower frequencies, usually accompanied by increased numbers of identical responses, were found in more chronic cases.

F-response shape showed rather less variation in MND patients than control subjects. About three quarters of the responses had only one or two negative peaks, compared with just over $50 \%$ in controls. Simple F-responses were particularly seen in recordings from wasted muscles, suggesting that a reduction in the number of available motor neurons and therefore a lesser chance of synchronous firing of multiple motor neurons was the main cause for the change in shape. F-responses in patients with CS differed little from controls, although in contrast to the MND group there was a tendency for the shape to be rather more complex than normal. Since there was only minimal lower motor neuron involvement of the hypothe- 
nar muscles, it is unlikely that complex polyphasic motor unit potentials resulting from denervation and reinnervation would be the major cause of the complex responses. Other possible mechanisms include in some cases an increase in the number of responding motor neurons with each stimulus, or desynchronisation of motor unit response latencies, both of which would lead to more variable overlapping of individual motor unit potentials in the Fresponse.

In conclusion the study has shown a reduction in the frequency of F-response production in patients with MND, which is related to the degree of muscle atrophy, and thus the loss of lower motor units. Despite the fall in overall F-response frequency there is a significant increase in the number of responses which repeat, and also in the frequency with which some of the repeating responses occur. These two features probably reflect a heightened degree of excitability within the spinal motor neuron pool due to upper motor neuron involvement. There was no significant change in overall F-response frequency in CS patients, although a small and significant increase in the number of repeating responses was observed in this group. The shape of F-responses was generally simpler than normal in MND patients, particularly in the presence of muscle wasting, while in CS patients, responses tended to have a normal or slightly more complex than normal shape.

The basic underlying processes of upper and lower motor neuron involvement are the same for both MND and CS. While there are clear quantitative differences between the MND and CS groups in respect of the F-response parameters examined in this study, it is questionable if these differences are of sufficient value on their own to differentiate between the two conditions for clinical diagnostic purposes, particularly in early stages of the disease. Nevertheless the finding of reduced numbers of $F$-responses or frequently repeating responses during the course of a peripheral nerve examination should alert the examiner to the possibility of the above pathologies.

\section{References}

1 Schiller HH, Stålberg E. F responses studied with single fibre EMG in normal subjects and spastic patients. J Neurol Neurosurg Psychiatry 1978;41:45-53.

2 Yates SK, Brown WF. Characteristics of the F response: a single motor unit study. J Neurol Neurosurg Psychiatry 1979;42:161-70.

3 Peioglou-Harmoussi S, Fawcett PRW, Howel D, Barwick DD. F-responses: a study of frequency, shape and amplitude in healthy control subjects. $J$ Neurol Neurosurg Psychiatry 1985;48:1159-64.

4 Fisher MA, Shahani BT, Young RR. Assessing segmental excitability after acute rostral lesions. 1 . The F-response. Neurology 1978;28:1265-71.

5 Swash M. Vulnerability of lower brachial myotomes in motor neurone disease. A clinical and single fibre EMG study. J Neurol Sci 1980;47:59-68.

6 Morimoto K. Clinical application of the F-wave. II Frequency of the F-wave. Kawasaki Med J 1980;6(1.2):49-64.

7 Wohlfart G. Collateral regeneration from residual motor nerve fibres in ALS. Neurology 1957;7:124-34.

8 Petajan JH. F-waves in neurogenic atrophy. Muscle Nerve 1985;8:690-96.

9 Wilkinson M. The morbid anatomy of cervical spondylosis and myelopathy. Brain 1960;83:589-617.

10 Mair WPG, Druckman R. The pathology of spinal cord lesions and their relation to clinical features in protrusions of cervical intervertebral discs. Brain 1953;76:70-91. 